\title{
Negative Life Events, Social Ties, and Depressive Symptoms for Older Adults in China
}

\author{
Hangqing Ruan ${ }^{1}$, Ke Shen ${ }^{2 *}$ and Feinian Chen ${ }^{1,2}$ \\ ${ }^{1}$ Department of Sociology, University of Maryland, College Park, MD, United States, ${ }^{2}$ School of Social Development and \\ Public Policy, Fudan University, Shanghai, China
}

\section{OPEN ACCESS}

Edited by:

Quishi Feng,

National University of

Singapore, Singapore

Reviewed by:

Herman Hay-ming Lo,

The Hong Kong Polytechnic

University, Hong Kong SAR, China

Qiang Li,

East China Normal University, China

*Correspondence:

Ke Shen

shenke@fudan.edu.cn

Specialty section:

This article was submitted to

Aging and Public Health,

a section of the journal

Frontiers in Public Health

Received: 12 September 2021

Accepted: 27 December 2021

Published: 20 January 2022

Citation:

Ruan H, Shen K and Chen F (2022) Negative Life Events, Social Ties, and

Depressive Symptoms for Older

Adults in China.

Front. Public Health 9:774434.

doi: 10.3389/fpubh.2021.774434
Although it is widely acknowledged that older adults who have gone through negative life events are more likely to develop depression, there is limited evidence on whether and which type of social ties moderate this perceived relationship. Based on 2016 and 2018 waves of Chinese Longitudinal Aging Social Survey (4,466 individuals, 8,932 observations), we apply linear fixed effects models and confirm that negative life events are associated with depressive symptoms for older adults (Coef. $=0.35$; 95\% Cls 0.11-0.61), and social ties are negatively associated with depression (Coef. $=-0.08$; $95 \%$ Cls -0.10 to -0.07 ). Our study further suggests that the association between negative life events and depressive symptoms is significantly moderated by friendship ties (Coef. $=-0.18,95 \% \mathrm{Cls}-0.30$ to -0.07 ), rather than family ties (Coef. $=-0.03,95 \%$ Cls -0.09 to 0.15$)$. Moreover, the buffering effects of friendship ties are more prominent for the less resilient and less privileged groups, namely male, rural, and less educated older adults. Our findings point to the importance of expanding and strengthening social networks for Chinese older adults in promoting their psychological health.

Keywords: negative life events, social ties, depressive symptoms, older adults, China

\section{INTRODUCTION}

World Health Organization (WHO) names depression among older adults as a key global public health concern (1), which leads to heightened disease burden, poor life quality, and high risk of suicide $(2,3)$. Unipolar depression or major depressive disorder, characterized by a persistent feeling of sadness or a lack of interest in outside stimuli, affected about $7 \%$ of the older adults worldwide in 2017 (1). In China, the age-standardized prevalence rate of depression saw a modest decline from 1990 to 2017 according to the Global Burden of Disease Study, that was as opposed to the global trend. However, it is noteworthy that the prevalence rate of depression for older adults aged over 55 years showed a clear upward trend over the same period and was much higher than that for younger adults (4), which was quite consistent with the global pattern. Another study based on the nationally representative survey in China revealed that 37.4 and $6.6 \%$ of older adults aged 61-75 reported depressive symptoms and severe depression, respectively, and the corresponding rates for those aged 75+ were even higher, at 40 and $9.7 \%$ in 2012 (5). Against the backdrop of accelerated aging process in China, depression has placed great strains on China's health care system and old-age support.

At present, the pathogenesis of depression has not been clearly identified, with genetic, socioeconomic, environmental, and behavioral factors potentially acting jointly (6-8). Among 
these factors, stress is acknowledged as an important risk factor for depression $(9,10)$. Negative life events, such as death of family members, severe illness, relationship crisis, and financial problems, pose tremendous stresses on older adults. A large amount of existing studies have demonstrated that experiencing negative life events are often associated with elevated risk of depression or increased depression severity for older adults in Western (11-13) and Asian contexts (14-16), and the magnitude of effects could vary by different types of life events and across various groups of population $(13,17)$. However, less attention has been paid to how to buffer the deteriorating impacts of negative life events $(12,18,19)$. Social support, as a key coping resource, has been documented to improve an individual's resilience and adaptability, and thus contribute to maintaining mental health in psychological research (20). Therefore, it is essential to incorporate social support to examine its moderating impact on the relationship between adverse life events and depression for older adults.

Social support is normally obtained from interpersonal relationships, both within and outside family. In the context of China and other East Asian societies with strong family network, older adults traditionally resort to family members for assistance and comfort in case of difficulties $(21,22)$. However, along with radical social transformation and massive migration, China has seen prominent family changes in recent decades, such as decline in average household size from 3.1 persons in 2010 to 2.6 persons in 2020, and large increase in one-person and one-generation households at old ages $(23,24)$. In this circumstance, support from outside household gets increasingly important and critical. The relative importance of family support and friend support in moderating the adverse impacts of negative life events needs to be further explored.

In this paper, based on the biennial panel data from 2016 and 2018 waves of Chinese Longitudinal Aging Social Survey (CLASS), a nationally representative elderly survey, we examine the association between negative life events and depressive symptoms for older adults, and more importantly, differentiate the moderating roles of family ties and friendship ties in the perceived association.

\section{LITERATURE REVIEW}

\section{Negative Life Events and Depression}

The stress process model provides a guiding framework for understanding the association between negative life events and depression. Based on stress process model, stressors that appear either in the form of disruptive life events or the more persistent hardships are related both to people's social and economic status and then to their health (25). Moreover, exposure to one stressor, regardless of whether it is an event or more chronic hardship, may lead over time to exposure to other, secondary, stressors, a process named as stress proliferation (26). Stress proliferation can result in people's lives becoming mired in clusters of stressors, some of which may contribute to cumulative adversity (27). Indeed, negative life events, such as family upheavals, illness, and injury, have been the focus of aging studies in the past decades because older adults often experience them at an elevated level and face heightened risk of stress proliferation. As such, negative life events has been considered as one of the most important stressors of depression for older adults $(12,28)$.

A large body of empirical studies have demonstrated that experiencing negative life events are associated with elevated risk of depression at old ages. For example, studies have repeatedly shown that family bereavement is closely related to depression at later life $(22,29-32)$. Some other studies have also found a positive relationship between depression and other negative events such as incidence of severe illness (11), relationship crisis and financial/work problem (33), or the total number of negative life events $(15,34,35)$. In 2002, a meta-analysis of 25 studies about negative life events and depression in 1980s and 1990s concluded that both the specific types of negative life events and total number of events is related to depression at a modest level $(r=0.15)$ for older adults (12). Another meta-analysis about 13 qualitative studies of depression in older ages also list negative life events as important risk factors of depression for older adults (36).

Similar results have been reported in Chinese context. One cross-sectional study with 385 community-dwelling older Chinse adults aged above 60 living in Singapore found that increased number of negative life events significantly increased older adults' depressive symptoms (16). Another two longitudinal studies in Hongkong about older adults aged 70 or above (260 older adults, 1992-1995) (14) and community-dwelling Chinese elderly aged 65 or above (2,630 older adults, 2001-2003) (17) also indicated that experiencing one or multiple events significantly increased the risk of depression for older adults.

In summary, a large amount of studies have demonstrated that negative life events are important risk factors of depression for older adults both in Western and Chinese context. However, less attention has been paid to whether and which type of social ties could moderate this perceived association.

\section{The Buffering Effect of Social Ties: Family Ties vs. Friendship Ties}

Social support is beneficial for mental health because it leads to "regular positive experience" and "a sense of predictability and stability in one's life situation, and a recognition of self-worth [(37), p. 311]." Moreover, social support has been documented as a key coping resource to buffer stresses from negative life events (20), but less is known about the structure or context of such support. It is essential to highlight the "contextual nature of social relation" in understanding the role of social support [(38), p. 84]. Social convoy model provides a theoretical framework to understand the dynamic contexts of social support for older adults. Based on social convoy model, individuals are "surrounded by supportive others who move with them throughout the life course" [(38), p. 84]. As for this study, social convoy model indicates that older adults are surrounded by social ties with dynamic function and of different types.

In East Asian context, with persistent Confucian culture, adult children have always been seen as the most important source of support for older adults $(21,22)$. Traditional family demographers have overwhelmingly emphasized the importance 
of family ties $(12,35)$ and empirical studies also found that living with an adult child could lower older adults depressive symptoms both in China and other East Asian countries like South Korea $(39,40)$. Some studies also argued that family ties can promote health only if the family relationship is harmonious $(41,42)$.

However, in recent years, the traditional multi-generational living arrangement among older adults has been declining in China (43). In this context, more and more studies turned to emphasize the importance of social ties outside the household (e.g., support from relatives or friends) (44). Friendship ties have been shown to be positively associated with older adult's happiness $(45,46)$, emotional well-being (47), self-esteem (48), and lowered suicide attempt (49). In Mainland China, it was also found that strong friendship ties is associated with lower depressive symptoms for rural older adults living alone (44).

Although existing literature highlights the importance of family and friendship ties, they put more attention to their direct effects on mental health and less to how they moderate the association between stressful life events and depression. The only exception is the a study by Chou and Chi (15), which showed that the family network, friendship networks, and confident relationships could ameliorate the deleterious effect of negative life events based on a cross-sectional sample of 411 older adults aged $60+$ in Hongkong. To the best of our knowledge, there is no study in Mainland China yet. Moreover, the study by Chou and Chi (15) did not compare the relative moderating roles of family networks and friendship networks. The differential buffering effects of family ties and friendship ties needs more rigorous examination.

\section{Subgroup Differences in Social Ties' Buffering Effect}

The meaning to and significance of social ties for different groups may be considerably different, and therefore, the buffering effects of social ties could vary by gender and socioeconomic status. For instance, studies suggest that the depressive symptoms among older adult is a little bit higher for women than men $(50,51)$, but male and female older adults use different coping strategies when facing stressful life events (52). Women are known as kinkeeper in the family (53) and have a wider and stronger social ties than men (54). In copying with negative life events in later life, women are more resilient (12), and on the contrary, men are more vulnerable in many situation.

Rural-urban differences have also been noted in China context (55). Numerous studies have examined the China's rural-urban divide in state policy, social and economic structure, health, and access to resources $(43,56-59)$. Rural older adults have higher depressive symptoms than those in urban area (55). But at the same time, rural older adults have less access to social security resources, which leads to more reliance on family and friend support in copying with stress (60). For example, one study in China found that the negative effect of disadvantaged living arrangement (e.g., living alone or living only with children) could be largely reduced by family and friendship ties in rural China, but this effect is less salient in urban China (44). The same logic can be applied to the educational difference in China. People with lower education often have higher level of depression $(50,51)$, but they are eligible to lower level of social security and have less access to coping resources (61), which make the less educated rely more on social ties when experiencing negative life events.

\section{RESEARCH HYPOTHESIS}

This paper focuses on the resilience of the older adults in China when experiencing negative life events and examines how social ties (including family ties and friendship ties) buffer the impacts of negative life events. Existing literature has documented that negative life events are important risk factors of depression in the later life (12). Therefore, we expect that experiencing negative life events would be detrimental to older adults' subjective well-being. In addition, we also expect that social ties (both family and friendship ties) are beneficial to older adults' subjective well-being.

Hypothesis 1: Experiencing negative life events will increase older adults' depressive symptoms.

Hypothesis 2: Social ties (including family ties and friendship ties) are negatively associated with older adults' depressive symptoms.

Based on the convoy model of social relations (38), we expect that social ties could buffer the association between negative life events and older adults' depressive symptoms:

Hypothesis 3: Older adults with stronger social ties have lower depressive symptoms than those with weaker social ties when experiencing negative life events.

In terms of the buffering effect of social ties, we will distinguish between the family ties and friendship ties. Previous studies have shown that family ties and friendship ties differ in the structure, quality, and function of relationship (44). But less is known about their relative importance in the contexts of negative life events for older adults in China. We expect that the buffering effect of family ties and friendship ties on the negative life events are different.

Hypothesis 4a: family ties play stronger roles in moderating the association between negative life events and depression than friendship ties.

Hypothesis 4b: friendship ties play stronger roles in moderating the association between negative life events and depression than family ties.

In addition, we also hypothesize the extent to which these ties ameliorate the perceived association differ across subpopulations. In general, we expect that the vulnerable and disadvantaged group benefits more from social ties. Considering older women are more resilient when facing negative life events, we expect that social ties may protect males more than females. We also expect social ties serve as more important protective barriers to the consequences of disruptive life events for rural and less educated older adults because they have less generous social security benefits and other coping repertoires.

Hypothesis 5a: The buffering effect of social ties on negative life events is stronger for the male than the female. 
Hypothesis 5b: The buffering effect of social ties on negative life events is stronger for older adults in rural area than those in urban area.

Hypothesis 5c: The buffering effect of social ties on negative life events is stronger for less educated older adults than the bettereducated.

\section{DATA AND METHOD}

\section{Data}

In this study, we use data from Chinese Longitudinal Aging Social Survey (CLASS) collected by the Institute of Aging Studies, Renmin University of China. The survey has been conducted for three waves in 2014,2016 , and 2018 , respectively. It is a nationally representative survey of older adults in China, covering 28 provinces, 134 counties, and 462 villages/neighborhoods in China (with 34 provinces and around 2,800 counties in total). The survey uses a multi-stage stratified probability sampling method, with county as the primary sampling unit and village/neighborhood as the secondary. Within each village or neighborhood, a random sample of the households is selected, and within each household, one older adult aged 60 and over is randomly selected as the survey respondent. It collects information on their demographic and socioeconomic characteristics, access to health and services, pension/retirement planning, cognitive abilities, and attitudes toward aging.

In this study, we use the biennial panel data of 2016 and 2018 waves of CLASS, as the definition of cognitive impairment (which is used to check if the respondent is suited for answering questions regarding depressive symptoms) has been changed since $2016^{1}$. The 2016 wave interviewed 11,471 older adults. Among them, 9,642 respondents ( $84 \%$ of the total) were traced in 2018 wave, while the rest either died or lost to follow up. We only keep the participants who joined both waves and get a sample size of 19,284 person-year observations. In these two waves, each respondent needed to answer eight cognition-related questions and only those who answered five or more questions correctly would proceed to answer questions regarding depressive symptoms and attitudes. Otherwise, the respondents were regarded as cognitively impaired and not suited to answer questions regarding depressive symptoms. As a result, 8,170 person-year observations $(42.37 \%)$ were excluded, and our sample reduced to 11,114 person-year observations $(5,557$ persons). We also drop the respondents with missing values in either wave (2,182 person-year observations, $11.3 \%)$, and most (84\%) of the missing is due to missing values on our dependent variable, depressive symptom ${ }^{2}$. Missing data has been a common challenge in health surveys that use self-report instruments. For

\footnotetext{
${ }^{1}$ In 2014 wave of CLASS, they use five cognition-related questions and only those answered at least three questions correctly were invited to finish the mental health and attitudes questions, but since 2016 three more questions were added and those who answered at least 5 questions correctly were able to participate in the mental health and attitudes questions.

${ }^{2} 1106$ person-year observations were missing in the CES-D, and another 728 person-year observations were dropped in Fixed-effects model because only one wave of the data has record in CES-D.
}

instance, among the 1,931 surgical patients surveyed in Canada, 351 (account for $18.2 \%$ of the total) did not fully complete the self-rated depression scale (62). The final sample size is 8,932 person-year observations (4,466 persons). We acknowledge that a missing rate of $11.3 \%$ is relatively high. But previous studies about depressive symptoms using CLASS data have shown similar (44) or even higher missing rate $(63,64)$, and their results were reasonable.

\section{Measurement \\ Depressive Symptoms}

Depressive symptoms were measured by using the Center for Epidemiologic Studies Depression (CES-D) scale (65). The scale involves the frequency of 9 items of symptoms which older adults have experienced in the past week: three items measuring feelings of positive affect (feeling happy, enjoying life, feeling pleasure), two items measuring feelings of negative affect (feeling lonely, feeling upset), two items measuring feelings of marginalization (feeling useless, having nothing to do), two items measured somatic symptoms having poor appetite, having trouble sleeping. The nine-item CES-D scale has been validated and widely used in assessing Chinese older adults' mental disorder $(64,66,67)$. We code the frequency with which the response had experienced each symptom in the past week as 0 (rarely or never), 1 (sometimes), 2 (often). The 9 items were summed up and a depression symptom score was created, ranging from 0 to 18 , with a higher score indicating more depressive symptoms.

\section{Negative Life Events}

It is a binary variable and is coded as 1 if the older adults experienced any of the following 9 items of negative experiences in the previous 12 months: (1) serious illnesses; (2) natural calamities; (3) death of spouse; (4) death of child/children; (5) death of other relatives or close friends; (6) serious financial loss; (7) serious illnesses of family members; (8) experiencing problems in a relationship with family members, relatives, or close friends; (9) experiencing accidents. It's to be noted that we also used different formats of coding of negative life events (e.g., the number of negative life events, or a 3 categorical variable of negative life events), and the results are consistent (results not shown $)^{3}$.

\section{Social Ties}

The Lubben Social Network Scale (LSNS) (68) is used in this paper to measure social ties for older adults. LSNS has been widely used in studies about social support for older adults in China $(35,44,69)$. The scale is constructed from a set of three questions evaluating family ties and a comparable three questions for friendship ties (1) "How many relatives (including family members)/friends do you see or hear at least once a month?, (2)

\footnotetext{
${ }^{3}$ For example, we use the number of negative life events (range from 0 to 4 ) as the key independent variable and the result shows that one more negative life events will lead to 0.265 points of increase in CES-D score $(p=0.010)$; compared with those did not experience negative life events, those who experience one event will have 0.338 points higher CES-D score, and those who experienced multiple $(\geq 2)$ events will be 0.419 points higher. But considering that the sample size of those who experienced multiple events is quite small (only about $1.87 \%$ of the sample), we use the binary negative life events in our following analysis.
} 
"How many relatives (including family members)/friends do you feel at ease with to talk about private matters?", and (3) "How many relatives (including family members)/friends do you feel close to such that you could call on them for help?." Response options range from none, one, two, three to four, five to eight, nine or more. We coded each item as 0 (none), 1 (one), 2 (two), 3 (three to four), 4 (five to eight), or 5 (nine or more). The three items were summed into a scale ranging from 0 to 15 for family ties and friendship ties, separately. Then the score of social ties, the sum of family and friendship ties, ranges from 0 to 30 .

\section{Demographic Variables}

We also control the following demographic variables in the analyses, including age, age squared, marital status (currently married or not), presence of grandchild, coresidence with adult children, self-reported health (range from 1 to 5 , the higher score, the better health), number of chronic diseases (range from 0 to 24 , which is measured by a list of 24 kinds of chronic conditions such as hypertension, cardiovascular diseases, diabetes, etc.) and index of functional limitations (range from 0 to 30, which is measured by how well the respondents could conduct 15 activities, such as dressing, eating, bathing, using the toilet, doing housework, etc.; the higher, the worse).

\section{Analytical Strategy}

We use the linear fixed effects regression model to conduct analyses. Let $y_{i t}$ denotes the CES-D score for individual $i$ in year $t(\mathrm{i}=1,2 \ldots \mathrm{N} ; \mathrm{t}=1,2)$.

$$
y_{i t}=X_{i t} \beta+u_{i}+\varepsilon_{i t}, \quad i=1,2,3 \ldots N ; t=1,2
$$

Where $X_{i t}$ are the time variant variables including negative life events and social ties (the social ties, family ties, and friendship ties are included in the model as continuous variables), and control variables for individual $i$ in year $t . u_{i}$ is the unobserved time-invariant effects which represents the individual heterogeneity, $\varepsilon_{i t}$ is the idiosyncratic errors which varies by individual and time.

Fixed effects model could correct for the endogeneity bias using the within transformation to eliminate timeinvariant unobservable characteristics (70). For instance, in this circumstance, older adult's early life experiences might jointly influence the risk of negative life events and depression at old ages. however, fixed effects model limits our analysis only to time variant variables in the analysis. All timeinvariant variables like gender and education would be removed in the demeaning process and are thus not included as control variables.

Using the fixed effects model, we first examine the relationship between negative life events and depressive symptoms controlling for all of the covariates mentioned above. We then include social ties as the key moderating variables, and differentiate the impact of family ties and friendship ties. lastly, we will also examine subsample differences by gender, rural/urban and education level attainment.

\section{RESULTS}

\section{Sample Description}

Descriptive statistics of key variables for the whole sample and for three groups of subsamples are presented in Table 1. Depressive symptom as measured by CES-D score in the full sample is 6.98 on average. Female older adults suffer more severe depressive symptoms than male counterparts. There are also rural-urban and educational disparities in depressive symptoms, with rural older adults and those with less than secondary education having higher CES-D score.

Overall, $14.7 \%$ of older adults have experienced at least one negative life event during the previous 12 months. Among the nine items, the three adverse life events the older adults are most likely to go through are death of other relatives or close friends, serious illnesses, and serious financial loss ${ }^{4}$. Female, rural, and the less educated older adults have slightly higher exposure to negative life events than male, urban, and the better-educated older adults, respectively.

The average score of social ties is 14.5 , and the strength of family ties is slightly stronger than the strength of friendship ties. The score of social ties is almost identical for males and females. Older adults living in rural area and having less than secondary education have weaker family ties and friendship ties than those living in urban area and having at least secondary education.

The average age of the older adults in our sample is about 69 years old, which is almost identical among all subgroups. $77 \%$ of older adults are currently married, $29.7 \%$ of them coreside with adult children, and $27 \%$ have grandchild presented in household, with these proportions varying a little among three groups of subsamples. The female, rural, and less educated older adults have worse health with higher number of chronic diseases and functional limitations.

\section{Association Between Negative Life Events, Social Ties, and Depressive Symptoms}

We examine the association between negative life events and depressive symptoms and address the moderating role of social ties on this perceived association. As shown in Model 1a in Table 2, after controlling for all the covariates, the CES-D score of those who have experienced at least one negative life events in the past 12 months is 0.350 higher than those who did not experience negative life events $(p=0.005)$. Moreover, the CES-D score decreases by 0.436 points with one standard deviation of increase in the score of social ties $(p<0.001)$, which means social ties are negatively associated with the severity of depression. These results are supportive of hypothesis 1 and 2.

In Model $1 \mathrm{~b}$, we add the interaction term of negative life events and social ties. We find that the deleterious effect of exposure to negative life events is significantly moderated by older adults' social ties (Coef. $=-0.081, p<0.001$ ), which confirms hypothesis 3 . To show the buffering effect of social ties more clearly, we present the predicted depressive symptoms by

${ }^{4}$ Due to space limitation, we do not present the detailed summary statistics of these nine negative life events. 
TABLE 1 | Descriptive statistics in total and subsamples, CLASS 2016 and 2018.

\begin{tabular}{|c|c|c|c|c|c|c|c|}
\hline Variable & Total & Male & Female & Rural & Urban & $\begin{array}{l}\text { Less than } \\
\text { Secondary }\end{array}$ & $\begin{array}{l}\text { Secondary } \\
\text { and above }\end{array}$ \\
\hline \multicolumn{8}{|l|}{ Dependent Variable } \\
\hline \multirow[t]{2}{*}{ CESD (range from 0 to 18 ) } & 6.98 & 6.93 & 7.04 & 7.20 & 6.80 & 7.11 & 6.80 \\
\hline & [2.91] & {$[2.87]$} & [2.95] & [2.78] & [2.99] & [2.82] & [3.01] \\
\hline \multicolumn{8}{|l|}{ Key independent variables } \\
\hline \multirow[t]{2}{*}{ Negative life events } & 0.15 & 0.14 & 0.15 & 0.16 & 0.14 & 0.15 & 0.14 \\
\hline & {$[0.35]$} & {$[0.35]$} & {$[0.36]$} & {$[0.36]$} & [0.35] & {$[0.36]$} & {$[0.35]$} \\
\hline \multirow[t]{2}{*}{ Social Ties (0-30) } & 14.50 & 14.50 & 14.49 & 14.42 & 14.56 & 14.21 & 14.89 \\
\hline & {$[5.25]$} & [5.39] & {$[5.08]$} & {$[5.45]$} & {$[5.07]$} & [5.29] & {$[5.15]$} \\
\hline \multirow[t]{2}{*}{ Family ties (0-15) } & 7.63 & 7.62 & 7.63 & 7.60 & 7.64 & 7.50 & 7.80 \\
\hline & {$[2.69]$} & {$[2.78]$} & {$[2.60]$} & {$[2.80]$} & {$[2.60]$} & {$[2.74]$} & {$[2.62]$} \\
\hline \multirow[t]{2}{*}{ Friendship ties (0-15) } & 6.87 & 6.88 & 6.87 & 6.81 & 6.92 & 6.71 & 7.10 \\
\hline & [3.06] & [3.13] & {$[2.98]$} & [3.16] & [2.98] & [3.09] & [3.01] \\
\hline \multicolumn{8}{|l|}{ Covariates } \\
\hline \multirow[t]{2}{*}{ Age } & 69.16 & 69.23 & 69.08 & 68.69 & 69.54 & 69.94 & 68.08 \\
\hline & {$[6.56]$} & [6.43] & {$[6.71]$} & {$[6.17]$} & [6.85] & [6.63] & [6.32] \\
\hline \multirow[t]{2}{*}{ Married (\%) } & 0.77 & 0.86 & 0.67 & 0.77 & 0.77 & 0.73 & 0.83 \\
\hline & {$[0.42]$} & {$[0.35]$} & {$[0.47]$} & {$[0.42]$} & [0.42] & {$[0.45]$} & {$[0.37]$} \\
\hline \multirow[t]{2}{*}{ Presence of grandchild (\%) } & 0.28 & 0.26 & 0.30 & 0.33 & 0.24 & 0.30 & 0.25 \\
\hline & {$[0.45]$} & {$[0.44]$} & {$[0.46]$} & {$[0.47]$} & [0.43] & {$[0.46]$} & [0.43] \\
\hline \multirow[t]{2}{*}{ Number of chronic diseases (0-24) } & 1.30 & 1.20 & 1.41 & 1.32 & 1.28 & 1.34 & 1.24 \\
\hline & {$[1.47]$} & [1.42] & [1.52] & {$[1.45]$} & [1.49] & {$[1.51]$} & [1.42] \\
\hline \multirow[t]{2}{*}{ Index of functional limitations (0-30) } & 0.82 & 0.68 & 0.97 & 0.86 & 0.79 & 0.92 & 0.67 \\
\hline & {$[2.31]$} & {$[2.14]$} & {$[2.49]$} & {$[2.42]$} & {$[2.23]$} & {$[2.40]$} & {$[2.18]$} \\
\hline \multirow[t]{2}{*}{ Self-reported health [1-5] } & 3.44 & 3.50 & 3.38 & 3.37 & 3.50 & 3.36 & 3.56 \\
\hline & {$[0.88]$} & {$[0.87]$} & {$[0.89]$} & {$[0.91]$} & {$[0.85]$} & {$[0.90]$} & {$[0.84]$} \\
\hline \multirow[t]{2}{*}{ Coresidence (\%) } & 0.30 & 0.28 & 0.32 & 0.33 & 0.27 & 0.31 & 0.28 \\
\hline & {$[0.46]$} & {$[0.45]$} & {$[0.47]$} & {$[0.47]$} & {$[0.44]$} & {$[0.46]$} & {$[0.45]$} \\
\hline Person-year observations & 8,932 & 4,732 & 4,200 & 3,997 & 4,935 & 5,182 & 3,750 \\
\hline
\end{tabular}

The mean values (or percentage) are presented, with standard deviation in the parentheses.

social ties and negative life events in Figure 1, which delivers three key information. First, the increase in the strength of social ties is associated with reduced depressive symptoms (the slope is negative either for dotted or solid lines). Second, the negative association between social ties and depression is stronger for those exposed to negative life events than for those who did not (the slope of dotted line is steeper than the slope of solid line). Third, when the social ties are weak, older adults who have experienced negative life events show more severe depressive symptoms than those who did not (a CES-D score of 9 vs. 8 when social ties score is 0 ). But when social ties get stronger, the gap in depressive symptoms becomes smaller, and is closed when the social ties score is around 20.

The results from Model 1a and Model $1 \mathrm{~b}$ indicate that social ties not only directly relate to lower depression severity, but also effectively moderate the shock from negative life events. But this does not tell us which type of social ties matters more. In model $2 \mathrm{a}$ and $2 \mathrm{~b}$, we distinguish the social ties into family ties and friendship ties to examine their differential impacts. In model $2 \mathrm{a}$, both family ties and friendship ties are significantly related to lowered depression severity for older adults, and this association is stronger for family ties (coef. $=-0.093, p<0.001$ ) than that of friendship ties (coef. $=-0.075, p<0.001$ ). In model $2 \mathrm{~b}$, we further add the interaction terms of negative life events with family ties and friendship ties, separately. It turns out that only friendship ties could effectively moderate the deleterious health consequences of negative life events (coef. $=-0.186, p$ $<0.001)$. This difference is clearly presented with the predicted depressive symptoms in Figure 2. In the left panel, regardless of the strength of family ties, the depressive symptom score for older adults exposed to negative life events is consistently higher than those who do not (the slopes of two lines are similar). But in the right panel of the graph, the gap in depressive symptoms between the groups with and without exposure to negative life events converges when friendship ties become stronger. These findings confirm hypothesis $4 \mathrm{~b}$ which argues for the stronger moderating roles of friendship than family ties.

The covariates in the models behave in expected directions. For example, depressive symptoms increase by age. Older adults who are currently married, have less chronic diseases 
TABLE 2 | The fixed effect models predicting depressive symptoms of chinese older adults, total sample, CLASS 2016 and $2018, N=8,932$.

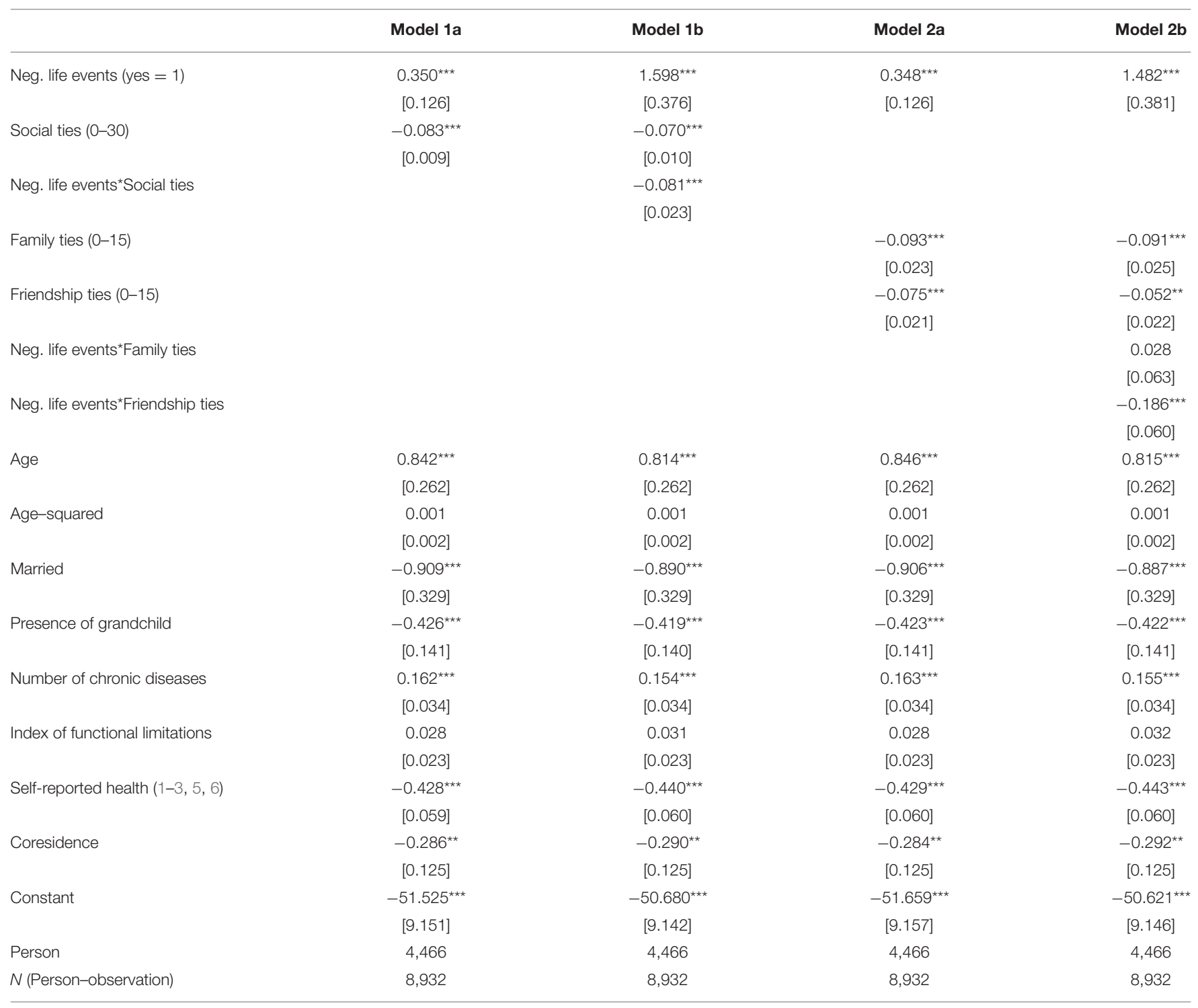

Note: The coefficient values included in the models are presented, standard errors in parentheses.

${ }^{* * *} p<0.01,{ }^{* *} p<0.05,{ }^{*} p<0.10$ (two-tailed tests).

and functional limitations, and report better health, have a lower score of depressive symptoms. Coresidence with an adult child and presence of grandchildren can lower the depressive symptoms.

Now we move to examine whether the buffering effect of social ties (family ties and friendship ties) differs by gender and socioeconomic status of the older adults. To do so, we split our data into three groups of subsamples: male vs. female, rural vs. urban, and less than secondary education vs. secondary education and above. We focus on the interaction effects of family/friendship ties with negative life events. As is shown in Table 3, for the total sample and each subsample, the association between negative life events and depressive symptoms is significantly moderated by friendship ties, rather than family ties, which further validates hypothesis 4b. More importantly, the moderating effect of friendship ties is stronger for male, rural, and less educated older adults. In other words, the coefficient of the interaction term of negative life events and friendship ties is significantly negative for male, rural and less-educated subsamples, but remains insignificant for female, urban and better educated subsamples. This finding confirms our hypothesis $5 a, 5 b$, and $5 c$, which means friendship ties play a more important role in moderating the association between disruptive life events and depression for less resilient and disadvantaged groups who have less alternative coping resources. 


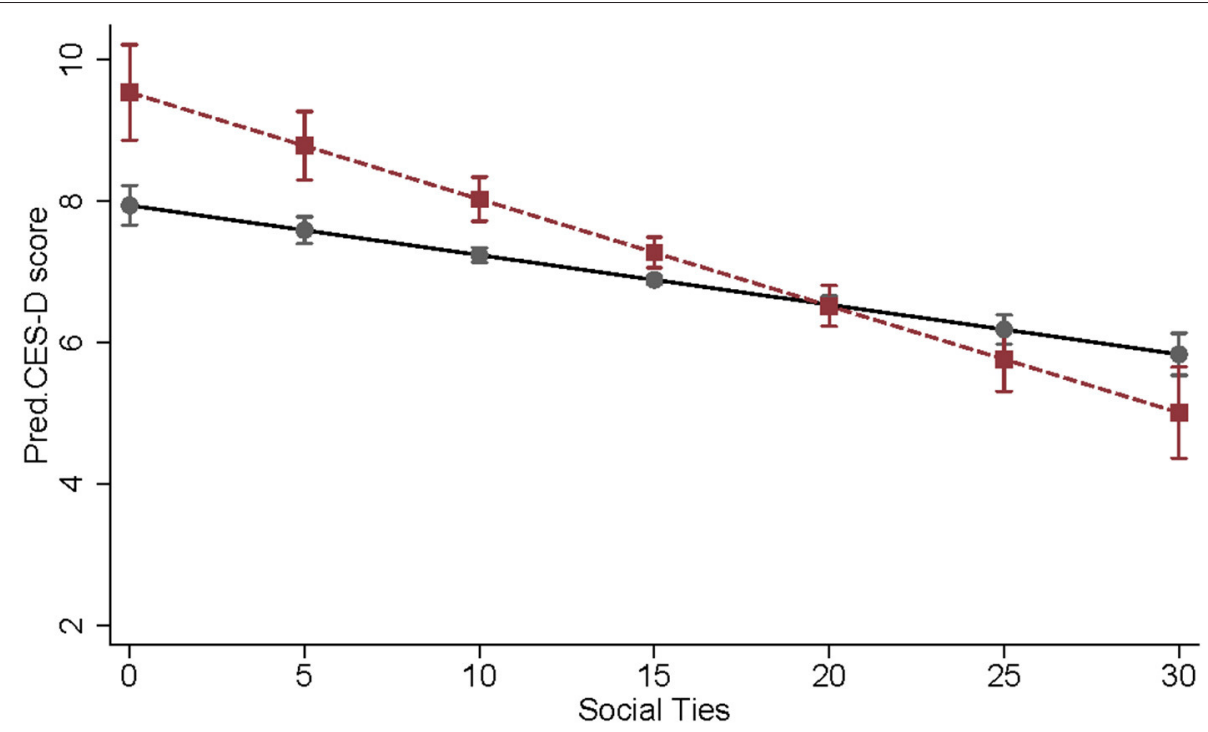

\section{$\longrightarrow$ No Events $\quad--\rightarrow---$ One or Multi. Events}

FIGURE 1 | Predicted depressive symptoms by social ties and negative life events (with 95\% confidence intervals), CLASS $2016-2016, \mathrm{~N}=8,932$.
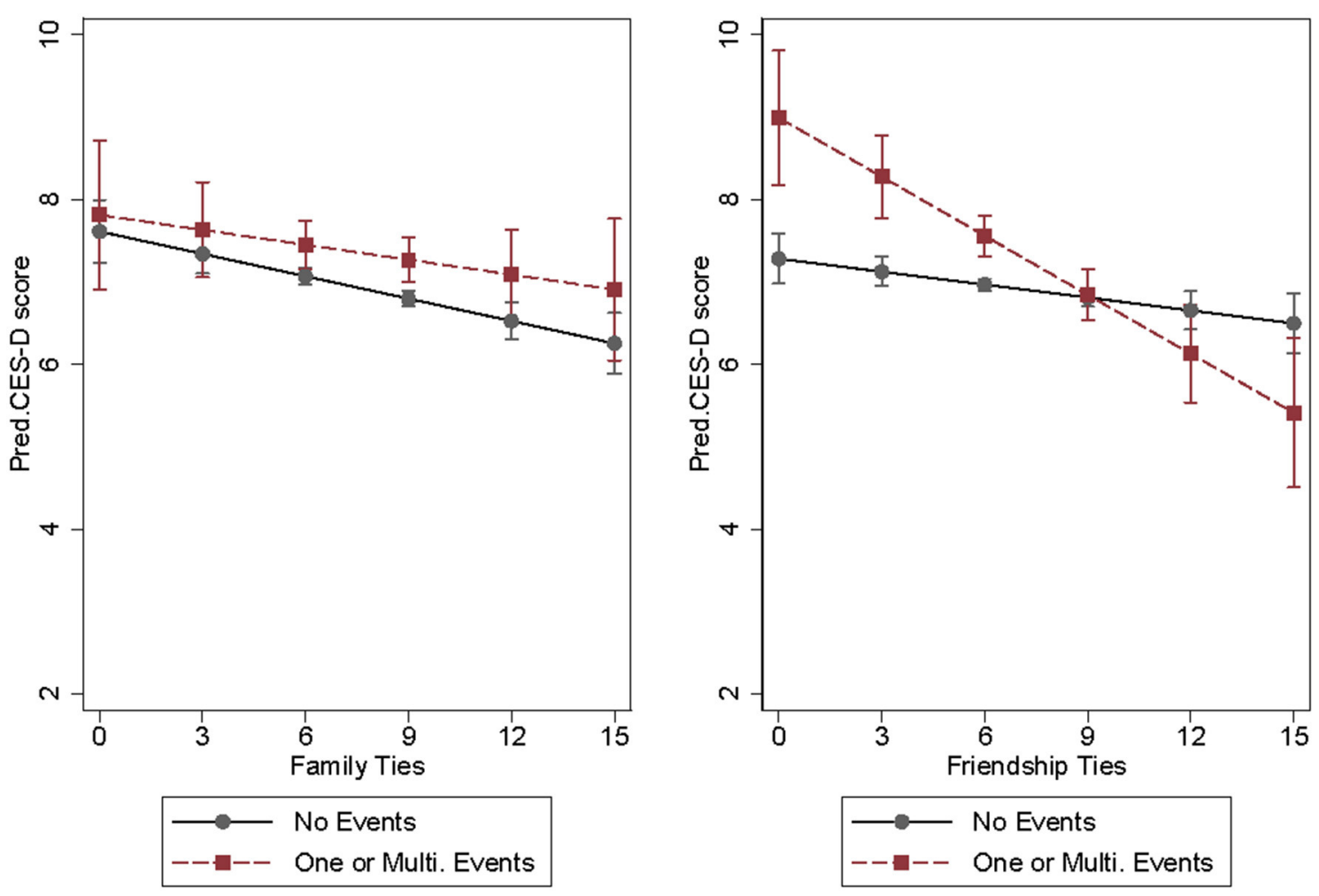

FIGURE 2 | Predicted depressive symptoms by family ties, friendship ties, and negative life events, CLASS 2016-2016, N = 8,932.

\section{DISCUSSION AND CONCLUSION}

Based on the nationally representative elderly survey in China, this paper examines the association between negative life events and depressive symptoms for older adults and explores how the perceived association is contingent on the strength of social ties. Our paper confirms that exposure to negative life events is an important risk factor of depression for older adults, echoing the 
TABLE 3 | The fixed effects models predicting depressive symptoms of Chinese older adults, by subsamples, CLASS 2016 and 2018, N=8,932.

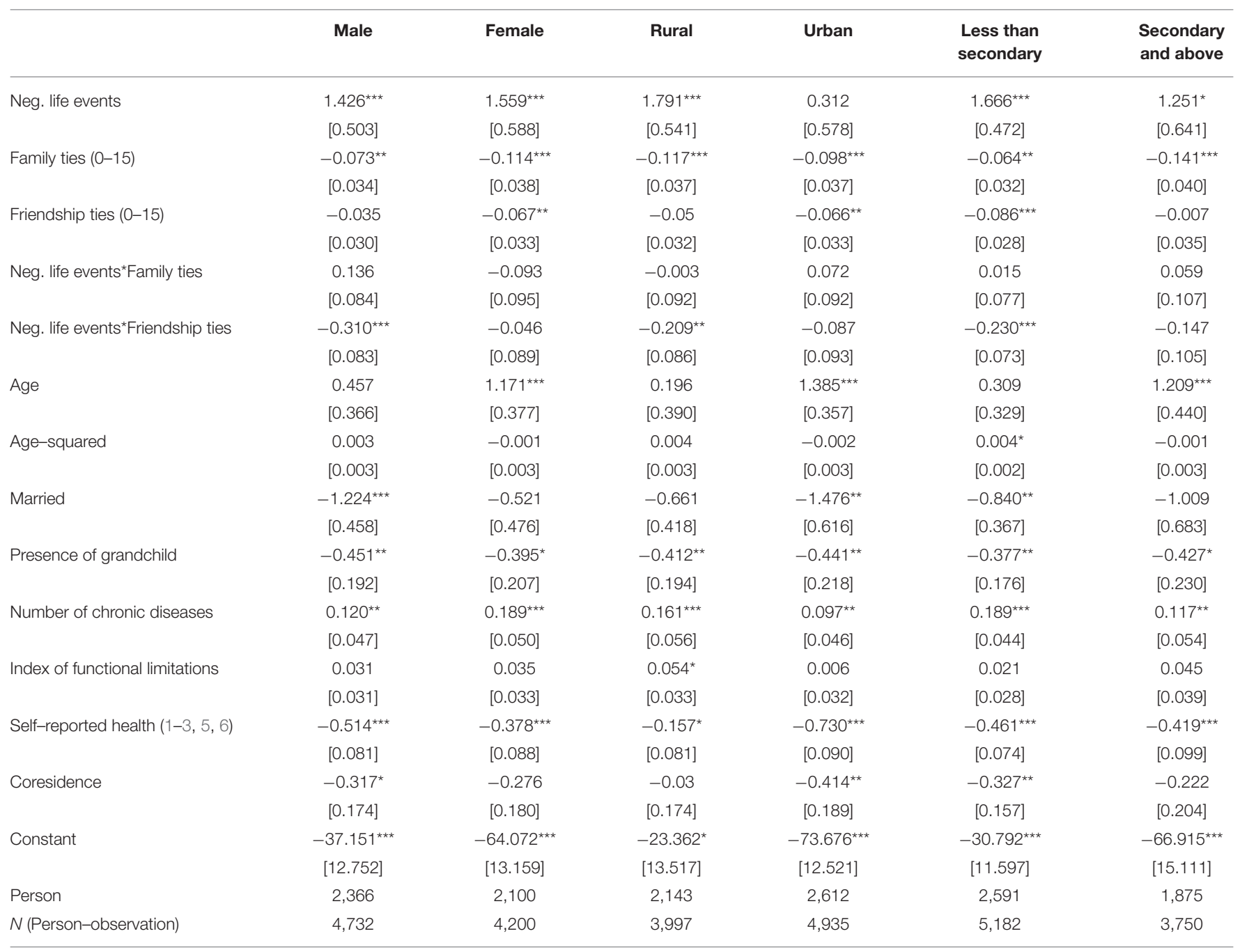

The coefficient values are presented, standard errors in parentheses.

${ }^{\star \star \star} p<0.01,{ }^{\star \star} p<0.05,{ }^{*} p<0.10$ (two-tailed tests).

previous findings for older adults in Singapore and Hong Kong of China $(14,16)$. Moreover, older adults with stronger social ties display less severe depressive symptoms than those with weaker social ties when experiencing negative life events. This finding in the context of mainland China enrich the evidence on the moderating roles of social ties, in support of the social convoy model ((38), p. 84).

More importantly, our paper expands on the current literature to compare the relative importance of family ties and friendship ties. On the one hand, both family ties and friendship ties are directly related to less severe depressive symptoms. On the other hand, the association between negative life events and depression is significantly moderated by friendship ties rather than family ties. Also, the moderating roles of friendship ties is more prominent for male, rural and less educated older adults, who are less resilient and have limited access to alternative coping resources. There might be two possible explanations for the stronger moderating roles of friendship ties as compared with family ties. First, the patriarchal culture strengthens family ties and intergenerational solidarity, but at the same time, it emphasizes the primary power and authority of male seniors and hinders intimate emotional interaction within household. Older adults might not be adapted to sharing painful and embarrassed feelings in case of stressful events with close family members $(71,72)$, thus friendship ties turn to be more important to cushion the shock. Second, the most common disruptive life event at old ages is death of family members. Family thus may become a pool where negative emotions and conflicts accumulate $(73,74)$, leading to a reduced buffering effect of family ties.

This paper also has several limitations. First, we code the exposure to negative life events as a dummy variable. Future studies could further differentiate the types of disruptive life events, such as family bereavement, severe illness, divorce, economic strains, and others. As such, they can figure out how social ties function to withstand the shock of various kinds of negative life events. Second, for each wave of the CLASS survey, 
self-reported negative life events are measured cross-sectionally and might be subject to recall bias. For instance, older adults with depressive symptoms tend to perceive usual experience more negatively and report it as a negative life event, leading to an overestimation of the association between negative life events and depression. In order to evaluate the severity of such bias, we conduct a sensitivity test by restricting the scope of negative life events to these less prone to recall bias such as the death of spouse/children/relatives, natural calamities, and accidents. The results remain robust, but we still need to be mindful of potential bias $^{5}$. Third, although we use fixed effects model to correct for the endogeneity bias resulting from time-invariant omitted variables, the endogeneity bias resulting from reversed causality might persist. For instance, severe depressive symptoms might lead to weakening friendship ties, such as, isolation from their friends, while have lesser impacts on family ties due to mutual obligations and commitments. Therefore, we should be cautious to attach a causal interpretation to our regression results. Future studies should use more rigorous design and longitudinal data to verify the time-varying effects of social ties on the association between negative life events and depression among the elderly.

Despite these limitations, our findings offer fresh evidence on the different roles of family and friendship support in moderating the association between stressful life events and depression in the context of mainland China, the society faced with accelerated aging process and intensified health concern. With heightened risk of encountering disruptive life events at old ages, the older adults are more susceptible to depression, which has become a major health challenge in China. Current policies often highlight the consolidation of family network to improve the well-being of older adults. For instance, the Central Committee of the Communist Party of China and the State Council jointly released a guideline addressing the issue of population aging in November 2021, that explicitly encourages adult children to co-reside or live

${ }^{5}$ Due to space limitation, the results are not shown in the paper and are available upon request.

\section{REFERENCES}

1. World Health Organization. Mental Health of Older Adults. (2017) 1-6 p. Retrieved from: https://www.who.int/news-room/fact-sheets/detail/mentalhealth-of-older-adults

2. Ferrari AJ, Norman RE, Freedman G, Baxter AJ, Pirkis JE, Harris MG, et al. The burden attributable to mental and substance use disorders as risk factors for suicide: findings from the Global Burden of Disease Study 2010. PLoS One. (2014) 9:e91936. doi: 10.1371/journal.pone. 0091936

3. Lépine JP, Briley M. The increasing burden of depression. Neuropsychiatr Dis Treat. (2011) 7(Suppl.):3-7. doi: 10.2147/NDT.S19617

4. Ren X, Yu S, Dong W, Yin P, Xu X, Zhou M. (2020). Burden of depression in China, 1990-2017: Findings from the global burden of disease study 2017. J Affect Disord. 268:95-101.

5. Qin X, Wang S, Hsieh CR. The prevalence of depression and depressive symptoms among adults in China: estimation based on a National Household Survey. China Econ Rev. (2018) 51:271-82. doi: 10.1016/j.chieco.2016. 04.001

6. Jeong Y, Kim JY, Ryu JS, Lee K, eun, Ha EH, et al. The associations between social support, health-related behaviors, socioeconomic close to their elderly parents. However, our study suggests a new and complementary perspective that highlights the importance of friendship ties for older adults' psychological health. Social ties outside the family, such as contact with and counseling from close friends, could act as a pivotal coping resource in relieving stresses from disruptive life events for older adults, in particular for those who are less resilient and have less access to alternative coping resources. Therefore, building and strengthening social network is as important as family network in promoting healthy aging in China.

\section{DATA AVAILABILITY STATEMENT}

The raw data supporting the conclusions of this article will be made available by the authors, without undue reservation.

\section{ETHICS STATEMENT}

Ethical review and approval was not required for the study on human participants in accordance with the local legislation and institutional requirements. Written informed consent for participation was not required for this study in accordance with the national legislation and the institutional requirements.

\section{AUTHOR CONTRIBUTIONS}

HR and FC analyzed data and interpreted the results. HR and KS drafted and revised the paper. All authors have read and approved the final version of the manuscript.

\section{FUNDING}

This work was supported by National Social Science Foundation of China (21\&ZD189) and National Science Foundation of China (72073032).

status and depression in medical students. Epidemiol Health. (2010) 32:e2010009. doi: 10.4178/epih/e2010009

7. Nugent NR, Tyrka AR, Carpenter LL, Price LH. Gene-environment interactions: early life stress and risk for depressive and anxiety disorders. Psychopharmacology. (2011) 214:175-96. doi: 10.1007/s00213-010-2151-x

8. Saveanu RV, Nemeroff CB. Etiology of depression: genetic and environmental factors. Psychiatric Clin N Am. (2012) 35:51-71. doi: 10.1016/j.psc.2011.12.001

9. Hammen C. Stress and depression. Annu Rev Clin Psychol. (2005) 1:293319. doi: 10.1146/annurev.clinpsy.1.102803.143938

10. Van Praag HM. Can stress cause depression? Prog Neuropsychopharmacol Biol Psychiatry. (2004) 28:891-907. doi: 10.1016/j.pnpbp.2004.05.031

11. Fiske A, Gatz M, Pedersen NL. Depressive symptoms and aging: the effects of illness and non-health-related events. J Gerontol Series B Psychol Sci Soc Sci. (2003) 58:320-8. doi: 10.1093/geronb/58.6.P320

12. Kraaij V, Arensman E, Spinhoven P. Negative life events and depression in elderly persons: a meta-analysis. J Gerontol Series B Psychol Sci Soc Sci. (2002) 57:87-94. doi: 10.1093/geronb/57.1.P87

13. Lefrançois R, Leclerc G, Hamel S, Gaulin P. Stressful life events and psychological distress of the very old: does social support have a moderating effect? Arch Gerontol Geriatr. (2000) 31:243-55. doi: 10.1016/S0167-4943(00)00083-2 
14. Chou KL, Chi I. Stressful events and depressive symptoms among old women and men: a longitudinal study. Int J Aging Human Dev. (2000) 51:27593. doi: 10.2190/VNJC-TQ4W-6T3R-6V9K

15. Chou KL, Chi I. Stressful life events and depressive symptoms: Social support and sense of control as mediators or moderators? Int J Aging Human Dev. (2001) 52:155-71. doi: 10.2190/9C97-LCA5-EWB7-XK2W

16. Lim ML, Lim D, Gwee X, Nyunt MSZ, Kumar R, Ng TP. Resilience, stressful life events, and depressive symptomatology among older Chinese adults. Aging Mental Health. (2015) 19:1005-14. doi: 10.1080/13607863.2014.995591

17. Chan D, Kwok A, Leung J, Yuen K, Choy D, Leung PC. Association between life events and change in depressive symptoms in Hong Kong Chinese elderly. J Affect Disord. (2012) 136:963-70. doi: 10.1016/j.jad.2011.08.031

18. Alexandrino-Silva CV, Alves TF, Tófoli LF, Wang YP, Andrade LH. Psychiatry - life events and social support in late life depression. Clinics. (2011) 66:2338. doi: 10.1590/S1807-59322011000200009

19. Dalgard OS, Dowrick C, Lehtinen V, Vazquez-Barquero JL, Casey P, Wilkinson G, et al. Negative life events, social support and gender difference in depression. Soc Psychiatry Psychiatr Epidemiol. (2006) 41:44451. doi: 10.1007/s00127-006-0051-5

20. Thoits PA. Mechanisms linking social ties and support to physical and mental health. J Health Soc Behav. (2011) 52:145-61. doi: 10.1177/00221465103 95592

21. Teerawichitchainan B, Pothisiri W, Long GT. How do living arrangements and intergenerational support matter forpsychological health of elderly parents? Evidence from Myanmar, Vietnam, and Thailand. Soc Sci Med. (2015) 136-137:106-16. doi: 10.1016/j.socscimed.2015.05.019

22. Wang J, Chen T, Han B. Does coresidence with adult children associate with better psychological well-being among the oldest old in China? Aging Mental Health. (2014) 18:232-9. doi: 10.1080/13607863.2013.837143

23. NBS. National Bureau of Statistics in China (NBS). Statistical Report of the Seventh Population Census in China. (2021). Available online at: http://www. stats.gov.cn/tjsj/tjgb/rkpcgb/qgrkpcgb/202106/t20210628_1818821.html

24. Shen K, Cai Y, Wang F, Hu Z. Changing society, changing lives: Three decades of family change in China. Int J Soc Welfare. (2021) 39:453-464.

25. Pearlin LI, Lieberman MA, Menaghan EG, Mullan JT. The stress process. J Health Soc Behav. (1981) 22:337-56. doi: 10.2307/2136676

26. Pearlin LI. The life course and the stress process: some conceptual comparisons. J Gerontol B Psychol Sci Soc Sci. (2010) 65:207-15. doi: 10.1093/geronb/gbp106

27. O'Rand AM. The precious and the precocious: understanding cumulative disadvantage and cumulative advantage over the life course. Gerontologist. (1996) 36:230-8. doi: 10.1093/geront/36.2.230

28. Glass TA, Kasl SV, Berkman LF. Stressful life events and depressive symptoms among the elderly: evidence from a prospective community study. J Aging Health. (1997) 9:70-89. doi: 10.1177/089826439700900104

29. Jadhav A, Weir D. Widowhood and depression in a cross-national perspective: evidence from the United States, Europe, Korea, and China. J Gerontol B Psychol Sci Soc Sci. (2018) 73:e143-e53. doi: 10.1093/geronb/gbx021

30. McGarry K, Schoeni RF. Widow(er) poverty and out-of-pocket medical expenditures near the end of life. J Gerontol B Psychol Sci Soc Sci. (2005) 60:S160-8. doi: 10.1093/geronb/60.3.S160

31. Sasson I, Umberson DJ. Widowhood and depression: new light on gender differences, selection, psychological adjustment. J Gerontol B Psychol Sci Soc Sci. (2014) 69:135-45. doi: 10.1093/geronb/gbt058

32. Su D, Wu XN, Zhang YX, Li HP, Wang WL, Zhang JP, et al. Depression and social support between China' rural and urban empty-nest elderly. Arch Gerontol Geriatr. (2012) 55:564-9. doi: 10.1016/j.archger.2012.06.006

33. Moos RH, Brennan PL, Schutte KK, Moos BS. Older adults' coping with negative life events: common processes of managing health, interpersonal, financial/work stressors. Int J Aging Human Dev. (2006) 62:39-59. doi: 10.2190/ENLH-WAA2-AX8J-WRT1

34. Ardelt M, Jeste DV. Wisdom and hard times: the ameliorating effect of wisdom on the negative association between adverse life events and well-being. J Gerontol B Psychol Sci Soc Sci. (2018) 73:137483. doi: 10.1093/geronb/gbw137

35. Chan A, Malhotra C, Malhotra R, Østbye T. Living arrangements, social networks and depressive symptoms among older men and women in Singapore. Int J Geriatr Psychiatry. (2011) 26:630-9. doi: 10.1002/gps.2574
36. Corcoran J, Brown E, Davis M, Pineda M, Kadolph J, Bell H. Depression in older adults: a meta-synthesis. J Gerontol Soc Work. (2013) 56:50934. doi: 10.1080/01634372.2013.811144

37. Cohen S, Wills TA. Stress, social support, and the buffering hypothesis. Psychol Bull. (1985) 98:310-57. doi: 10.1037/0033-2909.98.2.310

38. Antonucci TC, Ajrouch KJ, Birditt KS. The convoy model: explaining social relations from a multidisciplinary perspective. Gerontologist. (2014) 54:8292. doi: 10.1093/geront/gnt118

39. Do YK, Malhotra C. The effect of coresidence with an adult child on depressive symptoms among older widowed women in South Korea: an instrumental variables estimation. J Gerontol B Psychol Sci Soc Sci. (2012) 67:384-91. doi: 10.1093/geronb/gbs033

40. Li L, Liang J, Toler A, Gu S. Widowhood and depressive symptoms among older Chinese: do gender and source of support make a difference? Soc Sci Med. (2005) 60:637-47. doi: 10.1016/j.socscimed.2004. 06.014

41. Fiori KL, Antonucci TC, Cortina KS. Social network typologies and mental health among older adults. J Gerontol B Psychol Sci Soc Sci. (2006) 61:P2532. doi: 10.1093/geronb/61.1.P25

42. Lowenstein A. Solidarity-conflict and ambivalence: testing two conceptual frameworks and their impact on quality of life for older family members. J Gerontol B Psychol Sci Soc Sci. (2007) 62:S100-7. doi: 10.1093/geronb/62.2.S100

43. Chen F, Liu G. Population aging in China. In: Uhlenberg P, editor. International Handbook of Population Aging. Springer Netherlands (2009). p. 157-72. doi: 10.1007/978-1-4020-8356-3_8

44. Tang D, Lin Z, Chen F. Moving beyond living arrangements: the role of family and friendship ties in promoting mental health for urban and rural older adults in China. Aging Mental Health. (2019) 24:152332. doi: 10.1080/13607863.2019.1602589

45. Adams RG, Taylor EM. Friendship and happiness in the third age. In: Demir M, editor. Friendship and Happiness: Across The Life-Span and Cultures. Springer Netherlands (2015). p. 155-69. doi: 10.1007/978-94-017-9603-3_9

46. Demir M, editor. Friendship and Happiness: Across the Life-Span and Cultures. Springer Netherlands (2015). doi: 10.1007/978-94-017-9603-3

47. Lee GR, Ishii-Kuntz M. Social interaction, loneliness, and emotional well-being among the elderly. Res Aging. (1987) 9:459-82. doi: 10.1177/0164027587094001

48. Lee GR, Shehan CL. Social relations and the self-esteem of older persons. Res Aging. (1989) 11:427-42. doi: 10.1177/0164027589114002

49. Marver JE, Galfalvy HC, Burke AK, Sublette ME, Oquendo MA, Mann JJ, et al. Friendship, depression, and suicide attempts in adults: exploratory analysis of a longitudinal follow-up study. Suicide Life-Threat Behav. (2017) 47:660-71. doi: 10.1111/sltb.12329

50. Li D, Zhang D, jun, Shao J, jin, Qi X, et al. A meta-analysis of the prevalence of depressive symptoms in Chinese older adults. Arch Gerontol Geriatr. (2014) 58:1-9. doi: 10.1016/j.archger.2013.07.016

51. Zhang L, Xu Y, Nie H, Zhang Y, Wu Y. The prevalence of depressive symptoms among the older in China: a meta-analysis. Int J Geriatr Psychiatry. (2012) 27:900-6. doi: 10.1002/gps.2821

52. Rubio L, Dumitrache C, Cordon-Pozo E, Rubio-Herrera R. Coping: impact of gender and stressful life events in middle and in old age. Clin Gerontol. (2016) 39:468-88. doi: 10.1080/07317115.2015.1132290

53. Rosenthal CJ. Kinkeeping in the familial division of labor. J Marriage Fam. (1985) 47:965. doi: 10.2307/352340

54. Antonucci TC, Akiyama H. An Examination of Sex Differences in Social Support among Older Men and Women. Sex roles 17.11 (1987) 737-49 p. doi: $10.1007 / \mathrm{BF} 00287685$

55. Li LW, Liu J, Xu H, Zhang Z. Understanding rural-urban differences in depressive symptoms among older adults in China. J Aging Health. (2016) 28:341-62. doi: 10.1177/0898264315591003

56. Dorélien $\mathrm{A}, \mathrm{Xu} \mathrm{H}$. Estimating rural-urban disparities in self-rated health in China: impact of choice of urban definition. Demogr Res. (2020) 43:142960. doi: 10.4054/DemRes.2020.43.49

57. Hu Y, Li P, Martikainen P. Rural-urban disparities in age trajectories of depression caseness in later life: the China health and retirement longitudinal study. PLoS One. (2019) 14:e0215907. doi: 10.1371/journal.pone. 0215907 
58. Liu M, Zhang Q, Lu M, Kwon CS, Quan H. Rural and urban disparity in health services utilization in China [electronic resource]. Med Care. (2007) 45:767-74. doi: 10.1097/MLR.0b013e3180618b9a

59. Xu J, Wang J, King M, Liu R, Yu F, Xing J, et al. Rural-urban disparities in the utilization of mental health inpatient services in China: the role of health insurance. Int J Health Econ Manage. (2018) 18:37793. doi: 10.1007/s10754-018-9238-z

60. Liu D, Xi J, Hall BJ, Fu M, Zhang B, Guo J, et al. Attitudes toward aging, social support and depression among older adults: difference by urban and rural areas in China. J Affect Disord. (2020) 274:8592. doi: 10.1016/j.jad.2020.05.052

61. Fiske A, Wetherell JL, Gatz M. Depression in older adults. Annu Rev Clin Psychol. (2009) 5:363-89. doi: 10.1146/annurev.clinpsy.032408.153621

62. Shrive FM, Stuart H, Quan H, Ghali WA. Dealing with missing data in a multiquestion depression scale: a comparison of imputation methods. BMC Med Res Methodol. (2006) 6:57. doi: 10.1186/1471-2288-6-57

63. Guo Q, Bai X, Feng N. (2018) Social participation and depressive symptoms among Chinese older adults: a study on rural-urban differences. I Affect Disord. 239:124-30. doi: 10.1016/j.jad.2018.06.036

64. Wang Z, Yang H, Zheng P, Liu B, Guo Z, Geng S, et al. Life negative events and depressive symptoms: the China longitudinal ageing social survey. BMC Public Health. (2020) 20:968. doi: 10.1186/s12889-020-09119-0

65. Radloff LS. The CES-D scale: a self-report depression scale for research in the general population. Appl Psychol Meas. (1977) 1:385-401. doi: 10.1177/014662167700100306

66. Lin Z, Chen F. Evolving parent-adult child relations: location of multiple children and psychological well-being of older adults in China. Public Health. (2018) 158:117-23. doi: 10.1016/j.puhe.2018.02.024

67. Silverstein $\mathrm{M}$, Cong $\mathrm{Z}$, $\mathrm{Li} \mathrm{S}$. Intergenerational transfers and living arrangements of older people in rural China: consequences for psychological well-being. J Gerontol B Psychol Sci Soc Sci. (2006) 61:S256-66. doi: 10.1093/geronb/61.5.S256

68. Lubben J, Blozik E, Gillmann G, Iliffe S, Von Kruse WR, Beck JC, et al. Performance of an abbreviated version of the lubben social network scale among three European community-dwelling older adult populations. Gerontologist. (2006) 46:503-13. doi: 10.1093/geront/46.4.503
69. Leung YY, Teo SL, Chua MB, Raman P, Liu C, Chan A. Living arrangements, social networks and onset or progression of pain among older adults in Singapore. Geriatrics Gerontol Int. (2016) 16:693-700. doi: 10.1111/ggi.12539

70. Bollen KA, Brand JE. A general panel model with random and fixed effects: a structural equations approach. Soc Forces. (2010) 89:1-34. doi: 10.1353/sof.2010.0072

71. Kramer EJ, Kwong K, Lee E, Chung H. Cultural factors influencing the mental health of Asian Americans. West J Med. (2002) 176:227-31.

72. Taylor SE, Sherman DK, Kim HS, Jarcho J, Takagi K, Dunagan MS. Culture and social support: who seeks it and why? J Pers Soc Psychol. (2004) 87:35462. doi: 10.1037/0022-3514.87.3.354

73. Kramer BJ, Kavanaugh M, Trentham-Dietz A, Walsh M, Yonker JA. Predictors of family conflict at the end of life: the experience of spouses and adult children of persons with lung cancer. Gerontologist. (2010) 50:21525. doi: 10.1093/geront/gnp121

74. Kramer BJ, Boelk AZ, Auer C. Family conflict at the end of life: lessons learned in a model program for vulnerable older adults. J Palliat Med. (2006) 9:791-801. doi: 10.1089/jpm.2006.9.791

Conflict of Interest: The authors declare that the research was conducted in the absence of any commercial or financial relationships that could be construed as a potential conflict of interest.

Publisher's Note: All claims expressed in this article are solely those of the authors and do not necessarily represent those of their affiliated organizations, or those of the publisher, the editors and the reviewers. Any product that may be evaluated in this article, or claim that may be made by its manufacturer, is not guaranteed or endorsed by the publisher.

Copyright (C) 2022 Ruan, Shen and Chen. This is an open-access article distributed under the terms of the Creative Commons Attribution License (CC BY). The use, distribution or reproduction in other forums is permitted, provided the original author(s) and the copyright owner(s) are credited and that the original publication in this journal is cited, in accordance with accepted academic practice. No use, distribution or reproduction is permitted which does not comply with these terms. 